\title{
Solid-State Fermentation of Aspergillus niger to Optimize Extraction Process of Isoliquiritigenin from Glycyrrhiza uralensis
}

\author{
Jingwei Hao $\mathbb{D},,^{1,2}$ Dianwei Li, ${ }^{2}$ Yunrong Jing, ${ }^{2}$ Lei Zhang, ${ }^{2}$ Jiahui Liu, ${ }^{2}$ and Yubin Ji ${ }^{1}$ \\ ${ }^{1}$ Center for Life Science and Enviromental Science, Harbin University of Commerce, Harbin 150076, China \\ ${ }^{2}$ College of Life Sciences and Technology, Mudanjiang Normal University, Mudanjiang 157011, China
}

Correspondence should be addressed to Jingwei Hao; swxhjw@126.com and Yubin Ji; 120718961@qq.com

Received 29 July 2020; Revised 16 November 2020; Accepted 22 November 2020; Published 28 November 2020

Academic Editor: San Jun Shi

Copyright (c) 2020 Jingwei Hao et al. This is an open access article distributed under the Creative Commons Attribution License, which permits unrestricted use, distribution, and reproduction in any medium, provided the original work is properly cited.

We successfully extracted isoliquiritigenin from Glycyrrhiza uralensis via fermentation with Aspergillus niger and ultrasonicassisted extraction. In brief, we used A. niger fermentation to culture G. uralensis powder, and we optimized some key parameters such as reaction conditions of $\mathrm{pH}$, inoculation concentration of A. niger, fermentation time, and solid-liquid ratio. Based on a single-factor experiment, we utilized the response surface methodology (RSM) approach to optimize this extraction procedure. Using the RSM approach, optimized conditions of $\mathrm{pH}=3.694$, the solid-liquid ratio $=1: 2.155$, and the inoculation concentration of $A$. niger $=1466745$ were selected. Optimized conditions resulted in an extraction efficiency of $1.525 \mathrm{mg} / \mathrm{g}$. These results showed that the extraction of isoliquiritigenin was most affected by $\mathrm{pH}$ and then the time of fermentation and the solid-liquid ratio. Overall, the developed extraction technique yielded 5 times the amount of isoliquiritigenin when compared to traditional methods.

\section{Introduction}

Glycyrrhiza uralensis Fisch (G. uralensis) is a traditional medicinal plant used in China for a wide range of uses and a perennial legume herb with thick roots and rhizome. The roots and rhizome of G. uralensis are used as a tonic Chinese herbal medicine [1-5]. Qi invigorates the spleen, clears away heat, detoxifies and eliminates phlegm and coughs, and relieves pain and has synergistic effects with a range of drugs. Major components include triterpenoid saponins, flavonoids, and polysaccharides. Flavonoids are the component of $G$. uralensis in the most activity [6].

Isoliquiritigenin is a major dihydroflavonoid extracted from G. uralensis [7-9]. The molecular formula of isoliquiritigenin is $\mathrm{C}_{15} \mathrm{H}_{12} \mathrm{O}_{4}$, and it has a melting point of $198-200^{\circ} \mathrm{C}$ and forms as yellow needle crystals [10-13]. And the mature technology can be extracted and separated from plants, and its content is often used as an important index for the quality evaluation of G. uralensis [2-5]. Isoliquiritigenin has been demonstrated to have anticancer, antioxidation, anti-inflammatory, and antiviral effects and has been used in the treatment of asthma, diabetes, AIDS, and other diseases in humans [14-21].

Microbial fermentation is used to regulate the metabolism of reactants to ensure controlled production of products [22]. In the production of traditional Chinese medicines, microbial fermentation activity can be improved, abundance of components can be altered, and their toxicity can be reduced, thus offering opportunities for research and development of traditional Chinese medicines [23-25]. Aspergillus niger, as a large fungus, has the characteristics of vigorous growth, short fermentation cycle, and no toxin production. It is one of the safe strains certified by the Food and Drug Administration (FDA). It can secrete amylase, cellulase, glucosidase, and endoglucanase. Solid-state fermentation of A. niger is an effective method of biotransformation of Chinese medicinal materials by using enzymes produced by A. niger. It has the advantages of improving curative effect, reducing toxicity, and producing new active ingredients [26-28]. 
Response surface methodology (RSM) is a statistical method used for multivariable problems. RSM aids the design of tests and uses multiple quadratic regression equations to fit functional models between factors and response values [29].

In the current study, G. uralensis was used as the raw material to investigate the use of A. niger solid-state fermentation on the extraction efficiency of isoliquiritigenin. By modifying four properties of the extraction process, we aimed to develop an efficient process. Properties modified included time of inoculation, $\mathrm{pH}$ of fermentation, ratio of G. uralensis to extraction fluid liquid, and mass of $A$. niger inoculated. Optimum conditions for the extraction of isoliquiritigenin from G. uralensis were established via RSM.

\section{Materials and Methods}

\subsection{Experimental}

2.1.1. Chemicals and Reagents. G. uralensis was obtained from Mudanjiang Pharmaceutical Chain Co., Ltd. (Mudanjiang, China), and isoliquiritigenin standard (98\% purity) was obtained from the National Institute for the Control of Pharmaceutical and Biological Products (Beijing, China). Anhydrous ethanol was obtained from Nanjing Xingsha Chemical Co., Ltd., methanol from Puyang Wangda Chemical Co., Ltd., and acetonitrile from Jinan Century Tongda Chemical Co., Ltd. All other analytical-grade chemicals and solvents were obtained from Beijing Chemical Reagents Co. (Beijing, China).

2.1.2. Instrumentation and Analytical Conditions. Instrumentation used was as follows: a KQ-400DB ultrasonic cleaner (Shenzhen Keweida Ultrasonic Equipment Co., Ltd.), 98-1-b electronic temperature-regulating heating sleeve (Heze Shengbang Instrument Development Co., Ltd.), tp-213 electronic balance (Sartorius Instrument Equipment Co., Ltd.), and Waters 2695 HPLC (Waters Co., Milford, MA, USA). In addition, a HiQ sil-C18 reversed-phase column (4.6 mm $\times 250 \mathrm{~mm}, 5 \mu \mathrm{m}$, KYA TECH Corp., Tokyo, Japan) was used for chromatographic separation using a temperature vibration incubator (Shanghai Chuyi Instrument Equipment Co., Ltd).

Acetonitrile-water-acetic acid (32:68:0.5, v/v/v) was used as the mobile phase for HPLC analyses, with a flow rate of $1.0 \mathrm{~mL} / \mathrm{min}$, injection volume of $10 \mu \mathrm{L}$, and column temperature of $25^{\circ} \mathrm{C}$. Isoliquiritigenin was detected at an absorbance of $350 \mathrm{~nm}$.

The resultant calibration curve had formula $Y=3 \times 10^{7}$ $X+2 \times 10^{6}\left(R^{2}=0.9911\right)$, indicating a good linear fit for isoliquiritigenin ( $X$ : isoliquiritigenin concentration; $Y$ : peak area).

2.2. Measurement of Water Content of Glycyrrhiza. G. uralensis was ground into a fine powder using a grinder. Three samples of $1 \mathrm{~g} \mathrm{G}$. uralensis were weighed and then dried in an oven at $60^{\circ} \mathrm{C}$ for $24 \mathrm{~h}$ to calculate the moisture content. Water content was calculated as

$$
Y=\frac{a-b}{a} \times 100 \%,
$$

where $Y$ is the moisture content in $\% ; a$ is the initial wet weight; and $b$ is the dry weight. Water content of G. uralensis was $3.4 \%$.

2.3. Activation of A. niger: Preparation of the PDA Culture Medium. $150 \mathrm{~g}$ peeled potatoes were weighed, $750 \mathrm{~mL}$ was added, and the mixture was boiled. After boiling, potatoes were ground and filtered, and filter residues were discarded, while hot, filtered mixture was packed into small test tubes at $1 / 5$ th total volume. A. niger was inoculated in the resulting medium and cultured at $37^{\circ} \mathrm{C}$ in the incubator for 4 days.

\subsection{Ultrasonic-Assisted Extraction of A. niger following} Fermentation. $4 \mathrm{~g}$ of $G$. uralensis powder was weighed into a $100 \mathrm{~mL}$ conical flask, and distilled water was added at a ratio of solid to liquid and stirred to prepare the sterilized fermentation medium. To investigate the impact of $\mathrm{pH}$ on the extraction efficiency of isoliquiritigenin from G. uralensis, $\mathrm{pH}$ values of 3, 4, 5,6 , and 7 were selected. To investigate the effect of fermentation time on the extraction efficiency of isoliquiritigenin from G. uralensis, cultures were maintained for 2, 4, 6, and 8 days, respectively. To investigate the effect of material-to-liquid ratios on the extraction efficiency of isoliquiritigenin from G. uralensis, ratios of $1: 2,1: 3,1: 4,1: 5$, and $1: 6$ were selected. To investigate the effect of the inoculation count of $A$. niger on the extraction rate of isoliquiritigenin from $G$. uralensis, inoculation counts of $1 \times 10^{5}, 5 \times 10^{5}, 1 \times 10^{6}, 2 \times 10^{6}, 3 \times 10^{6}, 4 \times 10^{6}$, and $5 \times 10^{6}$ A. niger were investigated. Extraction efficiencies are expressed as the observed values of the target analytes.

2.5. Method of Extraction of Isoliquiritigenin following Fermentation. $100 \mathrm{~mL}$ of $75 \%$ ethanol was added to the fermented medium, mixed, and extracted using an ultrasonic water bath at $80^{\circ} \mathrm{C}$ for $0.5 \mathrm{~h}$. Then, filtrates were collected, and volumes were assessed to determine extraction rates.

2.6. Reference and Conventional Extraction Methods. Ethanol reflux extraction: $4 \mathrm{~g}$ of $G$. uralensis was weighed into a $250 \mathrm{~mL}$ round bottom flask, $100 \mathrm{~mL}$ of $75 \%$ ethanol solution was added, the solution was mixed, and reflux extraction was completed at $80^{\circ} \mathrm{C}$ in a water bath for $2 \mathrm{~h}$. The resulting mixture was filtered while hot, and the filtrate was collected.

Ultrasonic extraction: $4 \mathrm{~g}$ of $\mathrm{G}$. uralensis was weighed into a $250 \mathrm{~mL}$ round bottom flask, $100 \mathrm{~mL}$ of $75 \%$ ethanol solution was added, and the solution was mixed and extracted at $80^{\circ} \mathrm{C}$ in a water bath for $0.5 \mathrm{~h}$ using a sonicator. The resulting mixture was filtered while hot, and the filtrate was collected.

2.7. Optimization of Isoliquiritigenin Extraction by RSM. RSM was employed to optimize fermentation conditions using Box-Behnken data processing software (Design- 
Expert 7.0, Delaware, USA). Using the single-factor investigation approach, $\mathrm{pH}$, inoculation concentration of $A$. niger, and solid-liquid ratio were used as the independent variables and extraction efficiency of isoliquiritigenin as the dependent variable.

2.8. Statistical Analyses. To indicate the extraction efficiency of isoliquiritigenin, one-way ANOVA was used to determine the significant differences between experiments with different conditions. All statistical significances were accepted when $\alpha<0.05$. The results of HPLC analysis were expressed as means of extraction efficiency \pm SD. Data analyses were conducted in SPSS 22.0 software.

\section{Results and Discussion}

\subsection{Single-Factor Experimental Design}

3.1.1. Impact of Fermentation $\mathrm{pH}$ on UAE-Mediated Isoliquiritigenin Extraction from $G$. uralensis. The effect of fermentation $\mathrm{pH}$ on the extraction of isoliquiritigenin from G. uralensis is presented in Figure 1(a). When $\mathrm{pH}$ was 4, the rate of the extraction of isoliquiritigenin was relatively high and differed with $\mathrm{pH}$. As $\mathrm{pH}$ of fermentation increased, the extraction efficiency decreased. There were significant differences between different $\mathrm{pH}(P<0.05)$. These results were consistent with the previous work which has demonstrated that optimal $\mathrm{pH}$ of proteases of $A$. niger is approximately 4 .

3.1.2. Impact of Fermentation Time on Isoliquiritigenin Yield. The effect of fermentation time on the extraction rate of isoliquiritigenin from G. uralensis was investigated. As presented in Figure 1(b), there were significant differences between different fermentation times $(P<0.05)$. First, with the increase of fermentation time, the extraction rate of isoliquiritigenin increased. After 4 days of fermentation, the extraction of isoliquiritigenin was the highest. Subsequently, the extraction rate of isoliquiritigenin decreased with the increase of days. During the early stages of fermentation, growth and metabolism of $A$. niger would rely on available substrates such as the cell wall, thus promoting the release of isoliquiritigenin. Therefore, the concentration of isoliquiritigenin would have initially increased and then decreased as it might have been used as a substrate, thus decreasing its abundance.

\subsubsection{Impact of Solid-Liquid Ratios on Isoliquiritigenin Yield.} The extraction of isoliquiritigenin from G. uralensis was significantly impacted by the ratio of solid to liquid. As presented in Figure 1(c), the rate of extraction of isoliquiritigenin tended to decrease with increasing the ratios of solid to liquid. The results showed that there were significant differences among different solid-liquid ratios $(P<0.05)$. At the solid-1iquid ratio $=1: 2$, the rate of extraction of isoliquiritigenin was the highest, relatively.
3.1.4. Impact of Inoculation Concentration of A. niger on the Extraction of Isoliquiritigenin from G. uralensis. As presented in Figure 1(d), the extraction of isoliquiritigenin increased linearly with the increasing concentrations of $A$. niger. There were significant differences between different quantities of $A$. niger $(P<0.05)$. When the inoculation concentration was equal to $1 \times 10^{6}$, the extraction of isoliquiritigenin was the highest.

3.2. RSM Optimization of Fermentation Conditions. To further investigate interactions among fermentation conditions and optimize the extraction of isoliquiritigenin, RSM was applied. Experimental randomization was conducted as detailed in Table 1 in an effort to maximize the impact of unexplained variability on extraction efficiency. In total, we conducted 17 tests, with 5 replicates (runs 2, 7, 8, 12, and 15, Table 1), to estimate the pure error sum of squares.

The predicted $R^{2}$ value of 0.8235 was reasonably consistent with the adjusted $R^{2}$ value of 0.9482 , and the ratio of precision was 18.461 , thus indicating adequate precision (Table 2). Furthermore, our model had high $F$ values and low $P$ values $(P<0.0001)$ for two calculated responses. The $F$ value of 33.51 implies a $0.01 \%$ probability that is due to a random chance. For the result of RSM, any "Prob $>F$ " values $<0.0500$ are significant, whereas values $>0.1$ were not considered significant. Based on these criteria, the terms $B, C$, $A B, B^{2}$, and $C^{2}$ were considered significant (Table 3 ).

The "predicted $R$-squared" of 0.8235 is in reasonable agreement with the "adjusted $R$-squared" of 0.9482 , with a difference less than 0.2. "Adeq precision" measures the signal-to-noise ratio, where a ratio greater than 4 is desirable. The observed ratio of 18.461 indicates an adequate signal, suggesting that the model can be used to model the design space.

Results of the RSM analysis suggest that three independent variables were related as identified using the second-order polynomial equation: yield $(\mathrm{mg} / \mathrm{g})=1.49-0.016$ $A+0.054 B+0.20 C-0.095 A B+0.0025 A C-0.062 B C-0.049$ $A^{2}-0.21 B^{2}-0.33 C^{2}$.

The response surfaces for the impact of the independent variables on the average extraction efficiency of isoliquiritigenin are shown in Figure 2. Figures 2(a)-2(c) show the interaction of $\mathrm{pH}$, solid-liquid ratio, and inoculation concentration of $A$. niger. Results of the analysis resulted in point predictions of $\mathrm{pH}=3.694$, the solid-liquid ratio $=1: 2.155$, and the inoculation concentration of $A$. niger $=1466745$. Overall, the extraction efficiency was $1.525 \mathrm{mg} / \mathrm{g}$.

3.3. Verification Tests. Verification tests were conducted three times using point-prediction RSM conditions $(\mathrm{pH}=3.694$, solid-liquid ratio $=1: 2.155$, and inoculation concentration of $A$. niger $=1466745)$. The confirmatory analysis yielded a yield efficiency of $1.49 \pm 0.035 \mathrm{mg} / \mathrm{g}$.

3.4. Comparison of Extraction Methods. Two extraction approaches were used to compare extraction efficiencies: solid-state fermentation of $A$. niger and ultrasonic-assisted 


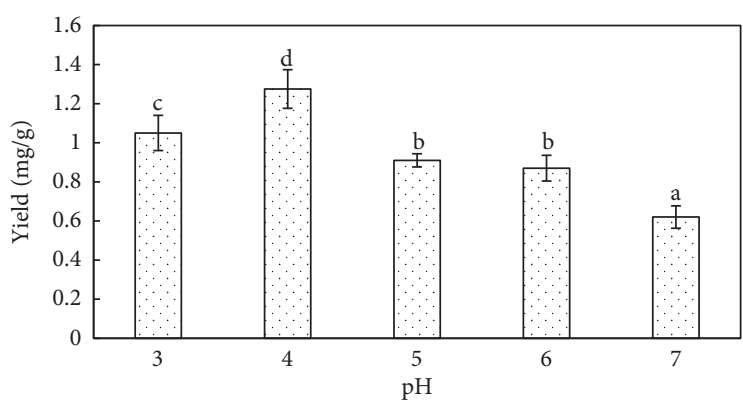

(a)

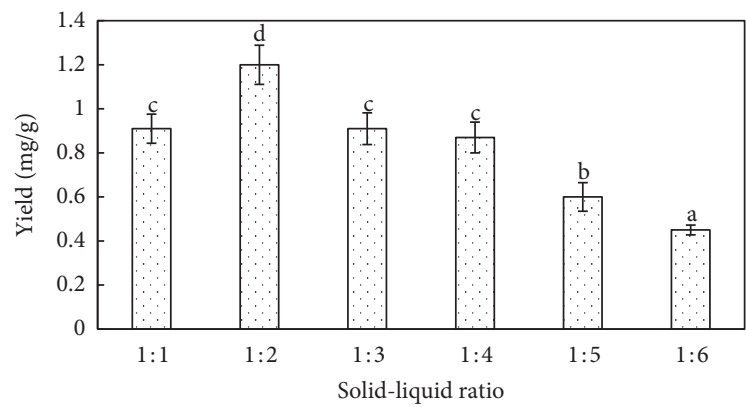

(c)

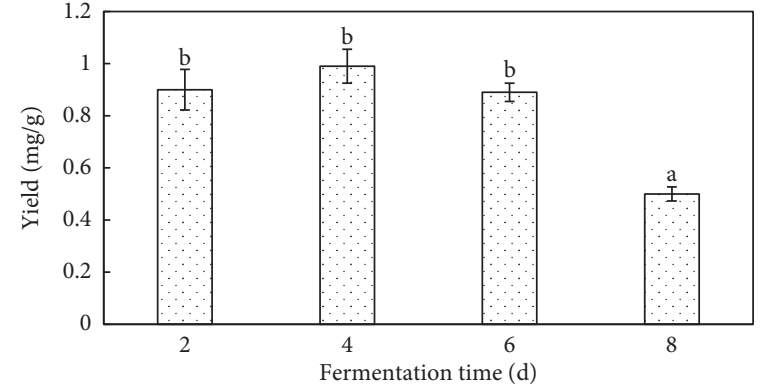

(b)

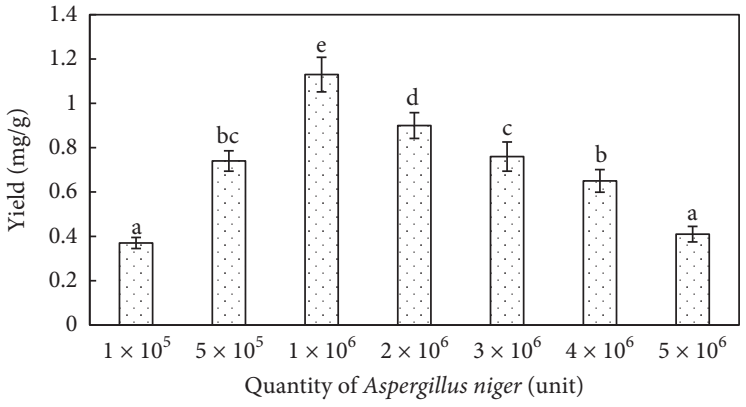

(d)

Figure 1: The extraction condition optimization. The following extraction parameters were utilized: (a) $0.4 \mathrm{~g}$ of the dried sample was combined with $75 \%$ ethanol aqueous solution. The inoculation count of $A$. niger was $2 \times 10^{6}$, solid-1iquid ratio was $1: 4$, fermentation time was 6 days, and inoculation temperature was $36^{\circ} \mathrm{C}$. (b) $0.4 \mathrm{~g}$ of the dried sample was combined with $75 \%$ ethanol aqueous solution. The inoculation count of $A$. niger was $2 \times 10^{6}$, the solid-1iquid ratio for this extraction was $1: 4$, pH was 5 , and the temperature was $36^{\circ} \mathrm{C}$. (c) $0.4 \mathrm{~g}$ of the dried sample was combined with $75 \%$ ethanol aqueous solution. Inoculation count of $A$. niger was $2 \times 10^{6}$, fermentation time was 6 days, $\mathrm{pH}$ was 5 , and temperature was set to $36^{\circ} \mathrm{C}$. (d) $0.4 \mathrm{~g}$ of the dried sample was combined with $75 \%$ ethanol aqueous solution, solid-1iquid ratio was $1: 4$, time of fermentation was 6 days, $\mathrm{pH}$ was 5 , and temperature was $36^{\circ} \mathrm{C}$. Different lowercase letters on the bars represent significant differences $(P<0.05)$ between treatments.

TABLe 1: Box-Behnken experimental design.

\begin{tabular}{lccc}
\hline Run & Factor $A(\mathrm{pH})$ & Factor $C$ (solid-liquid ratio) $(\mathrm{g} / \mathrm{mL})$ & Factor $B$ (inoculation amount of $A$. niger) \\
\hline 1 & 5 & 3 & $1.25 \times 10^{6}$ \\
2 & 4 & 2 & $1.25 \times 106$ \\
3 & 4 & 1 & $5 \times 10^{5}$ \\
4 & 3 & 1 & $1.25 \times 10^{6}$ \\
5 & 4 & 3 & $5 \times 10^{5}$ \\
6 & 5 & 2 & $2 \times 10^{6}$ \\
7 & 4 & 2 & $1.25 \times 10^{6}$ \\
8 & 4 & 2 & $1.25 \times 10^{6}$ \\
9 & 3 & 2 & $2 \times 10^{6}$ \\
10 & 5 & 2 & $5 \times 10^{6}$ \\
11 & 4 & 3 & $2 \times 10^{6}$ \\
12 & 4 & 2 & $1.25 \times 10^{6}$ \\
13 & 3 & 3 & $1.25 \times 10^{6}$ \\
14 & 4 & 1 & $2 \times 10^{6}$ \\
16 & 4 & 2 & $1.25 \times 10^{6}$ \\
17 & 5 & 1 & $1.25 \times 10^{6}$
\end{tabular}

extraction of isoliquiritigenin from G. uralensis. Extraction process was as follows: $4 \mathrm{~g}$ of the dried sample was mixed with $75 \%$ ethanol solution and refluxed for $2 \mathrm{~h}$ at a specific ratio of solution to raw material of $100 \mathrm{~mL}$. The overall yield was $0.167 \mathrm{mg} / \mathrm{g}$. The condition of the other method was similar to that outlined above, but ultrasonic extraction was used. Ultrasonic extraction methods destroy the external structure of the medicinal material at a specific frequency; therefore, the solvent can fully penetrate the medicinal material, resulting in a shortened extraction time and 
TABLE 2: Credibility analysis of regression equations.

\begin{tabular}{lc}
\hline Index marka & Extraction efficiency of lignans \\
\hline Std. dev. & 0.061 \\
Mean & 1.21 \\
C.V. $\%$ & 5.02 \\
PRESS & 0.2 \\
R-squared & 0.9773 \\
Adjusted $R$-squared & 0.9482 \\
Predicted $R$-squared & 0.8235 \\
Adeq precision & 18.461 \\
\hline
\end{tabular}

TABLE 3: Test of significance for the regression coefficient.

\begin{tabular}{|c|c|c|c|c|c|}
\hline Source & Sum of squares & $D f$ & Mean square & $F$ value & $P$ value \\
\hline Model & 1.11 & 9 & 0.12 & 33.51 & $<0.0001$ \\
\hline$A-\mathrm{pH}$ & $2.112 \times 10^{-3}$ & 1 & $2.112 \times 10^{-3}$ & 0.57 & 0.4739 \\
\hline$B$-solid-liquid ratio & 0.023 & 1 & 0.023 & 6.26 & 0.0408 \\
\hline$C$-inoculation amount of $A$. niger & 0.33 & 1 & 0.33 & 88.91 & $<0.0001$ \\
\hline$A B$ & 0.036 & 1 & 0.036 & 9.78 & 0.0167 \\
\hline$A C$ & $2.500 \times 10^{-5}$ & 1 & $2.500 \times 10^{-5}$ & $6.776 \times 10^{-3}$ & 0.9367 \\
\hline$B C$ & 0.016 & 1 & 0.016 & 4.23 & 0.0786 \\
\hline$A^{2}$ & 0.010 & 1 & 0.010 & 2.78 & 0.1391 \\
\hline$B^{2}$ & 0.18 & 1 & 0.18 & 50.04 & 0.0002 \\
\hline$C^{2}$ & 0.46 & 1 & 0.46 & 125.71 & $<0.0001$ \\
\hline Residual & 0.026 & 7 & $3.690 \times 10^{-3}$ & & \\
\hline Lack of fit & 0.011 & 3 & $3.708 \times 10^{-3}$ & 1.01 & 0.4759 \\
\hline Pure error & 0.015 & 4 & $3.676 \times 10^{-3}$ & & \\
\hline Cor total & 1.14 & 16 & & & \\
\hline
\end{tabular}

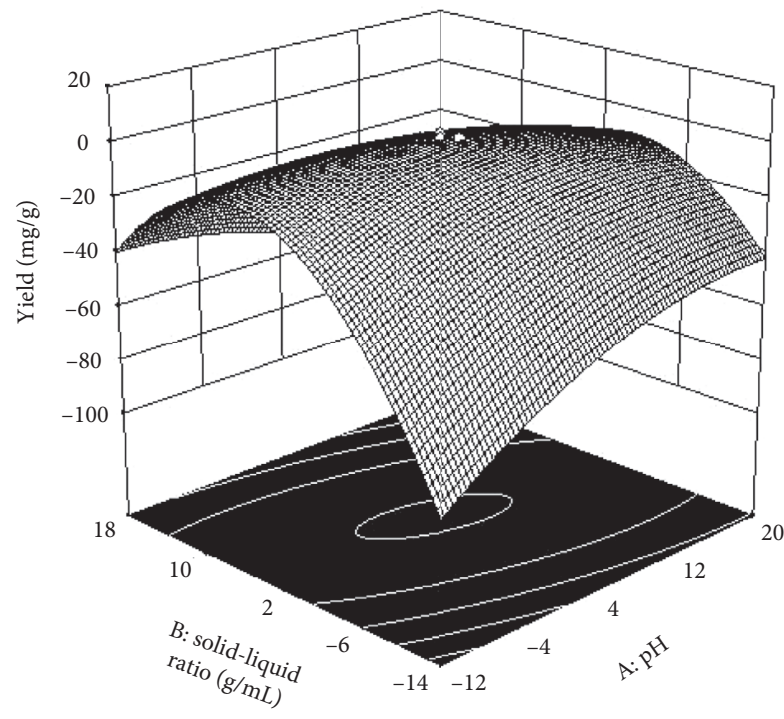

(a)

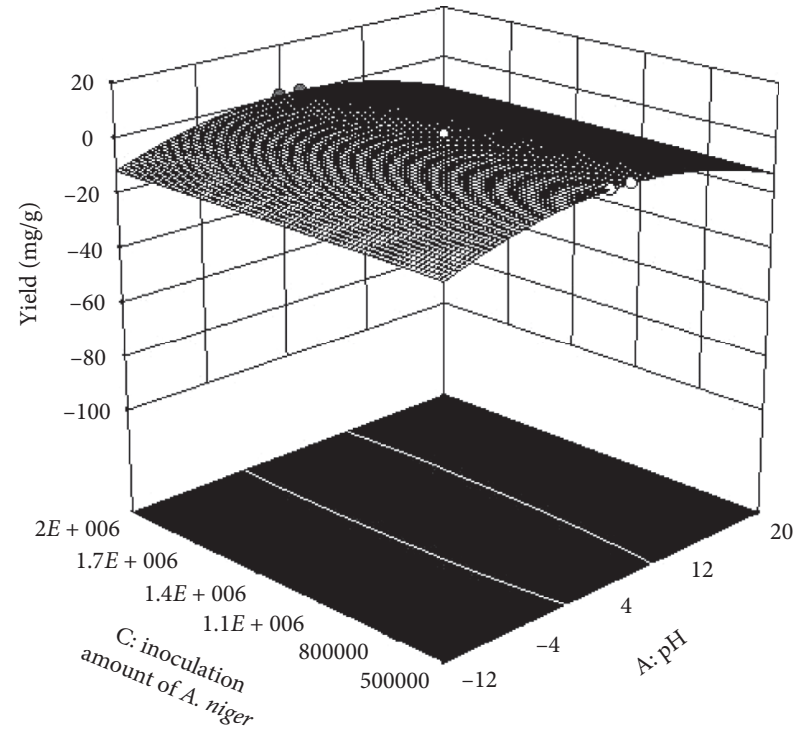

(b)

Figure 2: Continued. 


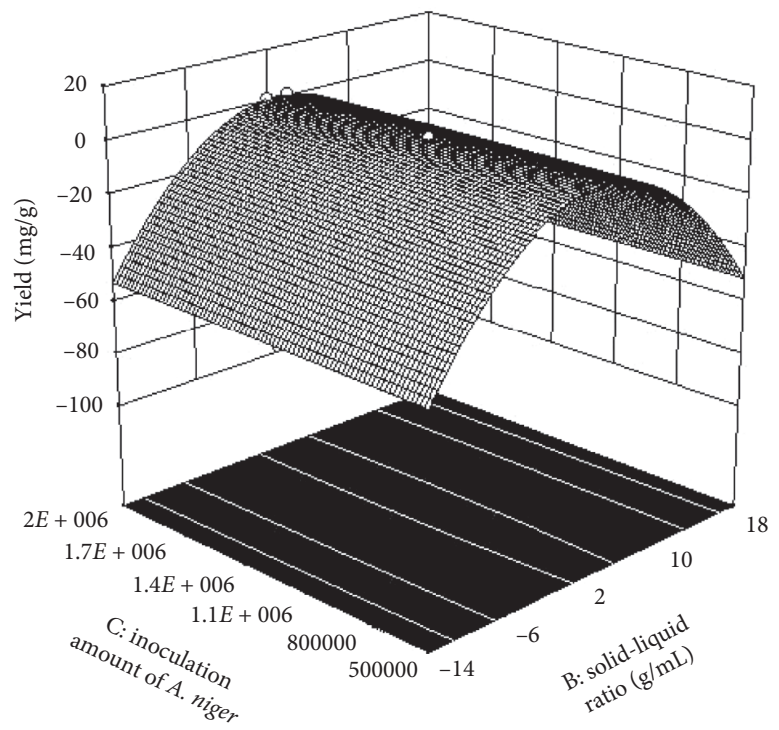

(c)

FIGURE 2: Response surface plots for the effect of variables on the average extraction efficiency of the target analyte. (a) Interaction of pH and solid-liquid ratio. (b) Interaction of $\mathrm{pH}$ and the inoculation concentration of A. niger. (c) Interaction of the inoculation concentration of A. niger and solid-liquid ratio.

improved extraction rate. In the present experiment, the extraction rate was $0.31 \mathrm{mg} / \mathrm{g}$.

\section{Conclusions}

In the current study, the fermentation of $A$. niger and ultrasonic-assisted extraction of isoliquiritigenin from G. uralensis were studied. A Box-Behnken design was employed to optimize extraction parameters. As demonstrated, the developed extraction process compared favorably $(5 \mathrm{x})$ with traditional extraction methods. To investigate the influence of extraction parameters, the RSM approach was utilized as employed in Box-Behnken software to optimize extraction procedures. In the analysis, $\mathrm{pH}$, inoculation concentration of $A$. niger, and solidliquid ratios were used as independent variables and extraction rate of isoliquiritigenin as the dependent variable. Optimized conditions were $\mathrm{pH}=3.694$, solidliquid ratio $=1: 2.155$, and inoculation concentration of A. niger $=1466745$ unit as the extraction efficiency of $1.525 \mathrm{mg} / \mathrm{g}$ was reached.

Because isoliquiritigenin in G. uralensis is present as combined and free portions, traditional solvent HRE methods of extraction only extract isoliquiritigenin from G. uralensis. The binding of isoliquiritigenin in G. uralensis is via aglycone; therefore, growth and metabolism of $A$. niger can release $\beta$-glucosidase, which can hydrolyze binding isoliquiritigenin into free isoliquiritigenin, thus increasing the rates of extraction. Results showed that fermentation time, solid-liquid ratio, $\mathrm{pH}$, inoculation concentration of $A$. niger, and other factors all influence the rate of extraction of isoliquiritigenin. $\mathrm{pH}$ influenced the rate of extraction of isoliquiritigenin from $G$. uralensis most, likely due to the fact that $\mathrm{pH}$ impacts growth and fermentation of $A$. niger. During growth and metabolism of $A$. niger, a number of enzymes are produced, and some enzymatic reactions occur. The cell wall of the plant tissue is mainly composed of cellulose, hemicellulose, pectin, and other macromolecules. Some specific enzymatic reactions can hydrolyze cellulose, pectin, and other macromolecules; therefore, components of interest are released and more easily extracted. During the second day of fermentation, mycelium of $A$. niger grew and began to produce spores. The enzymatic activity of secretions would have increased rapidly, and correspondingly, dissolution of isoliquiritigenin reached the maximum value. As the time increased, some cells might have begun to die or degrade extracted products.

\section{Data Availability}

The data used to support the findings of this study are included within the article.

\section{Conflicts of Interest}

The authors declare no conflicts of interest.

\section{Acknowledgments}

This work was supported by the Science and Technology Project of Mudanjiang (Z2018s074 and YQFK2020026), the Science Research Project of the Education Department of Heilongjiang Province (1353MSYQN009), and the Doctoral Research Fund of Mudanjiang Normal University (MNUB201907). 


\section{References}

[1] A. Tsuge, S. Hisaka, H. Hayashi, and M. Nose, "Effect of hot water extract of a glycyrrhizin-deficient strain of Glycyrrhiza uralensis on contact hypersensitivity in mice," Journal of Natural Medicines, vol. 74, no. 2, pp. 415-420, 2020.

[2] S. Park, S. Kwon, S. Lim, J.-K. Kim, K. Lee, and J. Park, "Licoricidin, an active compound in the hexane/ethanol extract of Glycyrrhiza uralensis, inhibits lung metastasis of 4T1 murine mammary carcinoma cells," International Journal of Molecular Sciences, vol. 17, no. 6, p. 934, 2016.

[3] A. Behdad, S. Mohsenzadeh, M. Azizi, and N. Moshtaghi, "Salinity effects on physiological and phytochemical characteristics and gene expression of two Glycyrrhiza glabra L. populations," Phytochemistry, vol. 171, Article ID 112236, 10 pages, 2020.

[4] J. Li, S. Liu, J. Wang, J. Li, J. Li, and W. Gao, "Gene expression of glycyrrhizin acid and accumulation of endogenous signaling molecule inGlycyrrhiza uralensis Fisch adventitious roots afterSaccharomyces cerevisiaeandMeyerozyma guilliermondiiapplications," Biotechnology and Applied Biochemistry, vol. 64, no. 5, pp. 700-711, 2017.

[5] J. Liu, L. Wu, S. Wei et al., "Effects of arbuscular mycorrhizal fungi on the growth, nutrient uptake and glycyrrhizin production of licorice (Glycyrrhiza uralensis Fisch)," Plant Growth Regulation, vol. 52, no. 1, pp. 29-39, 2007.

[6] X. Q. Li, L. M. Cai, J. Liu et al., "Liquiritin suppresses UVBinduced skin injury through prevention of inflammation, oxidative stress and apoptosis through the TLR4/MyD88/NF$\kappa \mathrm{B}$ and MAPK/caspase signaling pathways," International Journal of Molecular Medicine, vol. 42, pp. 1445-1459, 2018.

[7] Y. X. Jiang, Y. Y. Dai, Y. F. Pan et al., "Total flavonoids from radix Glycyrrhiza exert anti-inflammatory and antitumorigenic effects by inactivating iNOS signaling pathways," Evidence-based Complementary and Alternative Medicine, vol. 2018, Article ID 6714282, 10 pages, 2018.

[8] Y. H. Kim, D. E. Kim, and S. H. Lee, "Effects of $18 \beta$-glycyrrhetinic acid on fungal protease-induced airway inflammatory responses," Mediators of Inflammation, vol. 2018, Article ID 6461032, 12 pages, 2018.

[9] X. Liu, Q. Zhu, M. Zhang et al., "Isoliquiritigenin Ameliorates acute pancreatitis in mice via inhibition of oxidative stress and modulation of the Nrf2/HO-1 pathway," Oxidative medicine and cellular longevity, vol. 2018, Article ID 7161592, 12 pages, 2018.

[10] P. A. Ayeka, Y. Bian, P. M. Githaiga, and Y. Zhao, "The immunomodulatory activities of licorice polysaccharides (Glycyrrhiza uralensis Fisch.) in CT 26 tumor-bearing mice," BMC Complementary and Alternative Medicine, vol. 17, no. 1, p. 536, 2017.

[11] L. Teng, C. Kou, C. Lu et al., "Involvement of the ERK pathway in the protective effects of glycyrrhizic acid against the MPP+-induced apoptosis of dopaminergic neuronal cells," International Journal of Molecular Medicine, vol. 34, no. 3, pp. 742-748, 2014.

[12] M. J. Hsieh, C. W. Lin, S. F. Yang, M. K. Chen, and H. L. Chiou, "Glabridin inhibits migration and invasion by transcriptional inhibition of matrix metalloproteinase 9 through modulation of NF- $\kappa \mathrm{B}$ and AP-1 activity in human liver cancer cells," British Journal of Pharmacology, vol. 171, no. 2, pp. 3037-3050, 2014.

[13] T. Yoshida, M. Kobayashi, X. D. Li, R. B. Pollard, and F. Suzuki, "Inhibitory effect of glycyrrhizin on the neutrophildependent increase of R5 HIV replication in cultures of macrophages," Immunology \& Cell Biology, vol. 87, no. 7, pp. 554-558, 2009.

[14] S. Q. He, M. Gao, Y. F. Fu, and Y. N. Zhang, "Glycyrrhizic acid inhibits leukemia cell growth and migration via blocking AKT/mTOR/STAT3 signaling," International Journal of Clinical and Experimental Pathology, vol. 8, no. 5, p. 5175, 2015.

[15] H. L. Huang, M. J. Hsieh, M. H. Chien, H. Y. Chen, S. F. Yang, and P. C. Hsiao, "Glabridin mediate caspases activation and induces apoptosis through JNK1/2 and p38 MAPK pathway in human promyelocytic leukemia cells," PLoS One, vol. 9, no. 6, Article ID e98943, 2014.

[16] P. A. Ayeka, Y. Bian, P. .G. Mwitar et al., "Immunomodulatory and anticancer potential of Gan cao (Glycyrrhiza uralensis Fisch.) polysaccharides by CT- 26 colon carcinoma cell growth inhibition and cytokine IL-7 upregulation in vitro," BMC Complementary and Alternative Medicine, vol. 16, no. 1, p. 206, 2016.

[17] Y.-1. Chang, C.-1. Chen, C.-L. Kuo, B.-c. Chen, and J.-s. You, "Glycyrrhetinic acid inhibits ICAM-1 expression via blocking JNK and NF- $\kappa$ B pathways in TNF- $\alpha$-activated endothelial cells," Acta Pharmacologica Sinica, vol. 31, no. 5, pp. 546-553, 2010.

[18] H.-S. Kwon, S.-M. Oh, and J.-K. Kim, "Glabridin, a functional compound of liquorice, attenuates colonic inflammation in mice with dextran sulphate sodium-induced colitis," Clinical \& Experimental Immunology, vol. 151, no. 1, pp. 165-173, 2008.

[19] J. Chen, Z. Q. Zhang, J. Song et al., "18 $\beta$-Glycyrrhetinic-acidmediated unfolded protein response induces autophagy and apoptosis in hepatocellular carcinoma," Scientific Reports, vol. 8 , no. 1 , p. $9365,2018$.

[20] A. Guo, D. He, H. B. Xu, C. A. Geng, and J. Zhao, "Promotion of regulatory $\mathrm{T}$ cell induction by immunomodulatory herbal medicine G. uralensis and its two constituents," Scientific Reports, vol. 5, no. 1, p. 14046, 2015.

[21] A.-Y. Na, E.-J. Yang, J. M. Jeon, S. H. Ki, K.-S. Song, and S. Lee, "Protective effect of isoliquiritigenin against ethanolinduced hepatic steatosis by regulating the SIRT1-AMPK pathway," Toxicological Research, vol. 34, no. 1, pp. 23-29, 2018.

[22] M. Berovič, A. Pivec, T. Košmerl, M. Wondra, and Š. Čelan, "Influence of heat shock on glycerol production in alcohol fermentation," Journal of Bioscience and Bioengineering, vol. 103, no. 2, pp. 135-139, 2007.

[23] S. S. Marwaha, M. Puri, M. K. Bhullar, and R. M. Kothari, "Optimization of parameters for hydrolysis of limonin for debittering of kinnow Mandarin juice by Rhodococcus fascians," Enzyme and Microbial Technology, vol. 16, no. 8, pp. 723-725, 1994.

[24] F. U. Hartl, "Molecular chaperones in the cytosol: from nascent chain to folded protein," Science, vol. 295, no. 5561, pp. 1852-1858, 2002.

[25] Y. Chen, H. Ni, F. Chen, H. Cai, L. Li, and W. Su, "Purification and characterization of a naringinase fromAspergillus aculeatusJMUdb058," Journal of Agricultural and Food Chemistry, vol. 61, no. 4, pp. 931-938, 2013.

[26] X. Sun, Z. Liu, Y. Qu Y, and X. Li, "The effects of wheat bran composition on the production of biomass-hydrolyzing enzymes by Penicillium decumbens," Applied Biochemistry and Biotechnology, vol. 146, no. 1-3, pp. 119-128, 2008.

[27] G. S. Dhillon, S. K. Brar, M. Verma, and R. D. Tyagi, "Recent advances in citric acid bio-production and recovery," Food \& Bioprocess Technology, vol. 4, no. 4, pp. 505-529, 2011. 
[28] V. Kumar and P. Shukla, "Functional aspects of xylanases toward industrial applications," in Frontier Discoveries and Innovations in Interdisciplinary Microbiology, Springer India, Delhi, India, 2016.

[29] S. F. Sy Mohamad, F. Mohd Said, M. S. Abdul Munaim, S. Mohamad, and W. M. Azizi Wan Sulaiman, "Application of experimental designs and response surface methods in screening and optimization of reverse micellar extraction," Critical Reviews in Biotechnology, vol. 40, no. 1, pp. 341-356, 2020. 